\title{
Tactile perception and Friction-induced Vibrations: Discrimination of similarly patterned wood-like surfaces
}

\author{
A. Jessica DACLEU NDENGUE, B. Ilaria CESINI, C. Jenny FAUCHEU, D. Eric ChATELET, E. \\ Hassan ZAHOUANI, F. David DELAFOSSE, G. Francesco MASSI
}

\begin{abstract}
The tactile perception of a surface texture is mediated by factors such as material, topography and vibrations induced by the sliding contact. In this paper, sensory characterizations are developed together with topographical and tribo-tactile characterizations to relate perceived features with objective measurements of tribological and dynamic signals. Two sets of surface samples are used in this study: the first set is made of a commercial floor covering tiles that aim at counter-typing natural wood flooring, with both a visual and a tactile texture mimicking wood. A second set is custom-made by replicating the first set using a plain purple polyurethane resin. The comparison between tribo-tactile signals and sensory analysis allowed the identification of objective indices for textures with slight topographical differences. Even though the topography of the replicated samples is the same as their corresponding commercial products, the fact that the material is different, induces differences in the contact and vibrational parameters. This in turn modifies the discrimination performances during the sensory experiment. Tactile characteristics collected during sensory procedures are found to be in agreement with objective indices such as friction coefficients and induced vibrations.
\end{abstract}

Index Terms - Friction-induced vibrations, Tactile perception, Tactile tribology, Textured materials

\section{INTRODUCTION}

Touch is one of the senses we rely on for various daily tasks. It provides accurate information about material and surface properties [1], and is directly associated with the "touch feeling". Interest in tactile perception has been growing during recent decades for many reasons: the need for developing human-machine interfaces, virtual reality equipment, touchscreens, etc. The human hand is a very complex system, and a huge amount of literature can be found in the different disciplines (like biology, psychology, neurophysiology, biomechanics, etc.), that is directly related to hand functions and touch. When a finger scans the surface of an object, the sliding contact generates vibrations that propagate in the skin and activate the mechanoreceptors. The friction-induced vibrations are essential to the perception of surface properties

- J. Dacleu Ndengue, J. Faucheu and D. Delafosse are with Ecole des Mines de Saint-Etienne, Centre SMS, CNRS LGF UMR 5307, 158 Cours Fauriel, 42023 saint-Etienne, France; (e-mail: Jessica.dacleu@emse.fr; faucheu@emse.fr; david.delafosse@emse.fr)

- I. Cesini and F. Massi are with the Department of Mechanics and Aerospace Engineering, University of Rome "La Sapienza", via Eudossiana 18, 00154, Rome, Italy (e-mail: ilariacesini91@gmail.com

;francesco.massi@uniroma1.it)

- E. Chatelet is with Laboratoire de Mécanique des Contacts et Structures, UMR 5259, INSA Lyon, Villeurbanne, France (e-mail: eric.chatelet@insalyon.fr)

- H. Zahouani is with Laboratoire de tribologie et de dynamique des systèmes, UMR 5513, Ecole centrale Lyon, Ecully France (e-mail: hassan.zahouani@ec-lyon.fr) such as roughness and softness, which are the main features for the discrimination of surface texture [2-3-4-5-6]. The mechanoreceptors transduce the stress state (mechanical signals) into electrical inputs (electrical signals), which are sent to and coded by the brain. Tactile sensing involves different types of units, made of afferent fibers connected to end organs such as Merkel disk, Meissner corpuscles, Ruffini endings and Pacinian corpuscles (Fig.1). They are located at different depths in the skin, ranging from the epidermis (Meissner corpuscles) to the subcutaneous fat layer below the dermis [8-9].

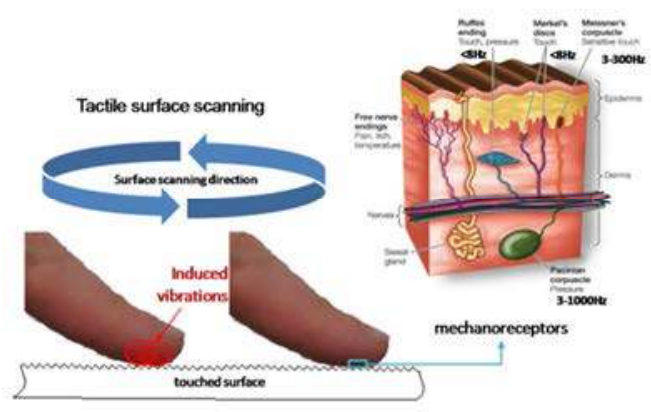

Fig.1. Induced vibrations when scanning a surface and the mechanoreceptors with their frequency range of activation. Reproduced from [7] with permission from the publisher Sinauer Associates Inc.

Previous work [10-11-12] has shown some correlations between surface properties and friction-induced vibrations when a fingertip scans a surface. By mapping the 
spatial distributions of vibrations propagating in the skin of the dorsal region of the hand during different manipulation tasks and analyzing their intensity and energy content, it was possible to obtain information regarding the type of interaction and manipulated object's characteristics [13]. The detection and reproduction of vibration signals caused by a tool-surface contact have been employed to create virtual textures [14]. The frequency distribution of the vibrations [9] was correlated with the "duplex perception mechanism" [5-12]. The range of the contact pressure used by humans for exploring surfaces [15] has been correlated with the stability of the vibration amplitude with the load [11]. Recently, different tactile descriptors (fibrous, relief, blocking) have been associated with objective indices derived from measurements of the induced vibrations and friction coefficients [16].

The tactile perception of wood-based materials has previously been the subject of research. The tactile attributes of wood and wood-based composites were investigated in [17]. The most significant differences between the samples were found for roughness and for the descriptors reliable, natural and solid. Another study [18] compared samples derived from objects of biological origin (human skin, wood), artifacts of biological origin (natural fabric, plushie) and objects made of non-biological materials (glass, metal). They found a strong correlation between the perceived attributes and the frictional properties of the materials.

This paper aims at highlighting the essential contribution of friction-induced vibrations to the perception of textures and suggests the possibility identifying objective measurable indices that can give insights into the discrimination strategy adopted by humans. Such indices could contribute to the characterization of the mechanical stimuli that are at the first stage of human perception, which is a necessary condition for recovering, understanding and reproducing "tactile signals". The influence of the texture of wood-like surfaces on individual perception is investigated.

\section{MATERIAL AND METHODS}

\subsection{Surface Samples}

Two sets of samples were used in this work, in order to study the tactile effects elicited from texture and from material. The first set was made of commercial floor covering tile that aims at simulate natural wood flooring, with both visual and tactile texture mimicking wood. A second set of samples was custom-made by replicating the first set of samples, using a plain purple polyurethane resin.

\subsubsection{Wood Countertype (WC) samples}

This first set contained 9 rectangular $\left(10^{*} 15.2 \mathrm{~cm}^{2}\right)$ samples cut from artificial wood flooring tiles. The "wood" flooring tiles are commercial products made of vinyl tiles with a wood-like visual printing (Fig. 2a) and an inksurface topography similar to wood grains and fibers (Fig. 2b). Since visual perception is not the aim of this study, only tactile texture will be discussed in this paper.

\subsubsection{Replica samples}

The second set contained 9 rectangular $\left(10^{\star} 15.2 \mathrm{~cm}^{2}\right)$ samples. These samples were custom-made by replicating each sample from the WC set using silicon molds and polyurethane purple resin (Fig. 2c). This technique made it possible to replicate the topography of the samples but the material used in the (R) sample set was different from the one used in the (WC) sample set.

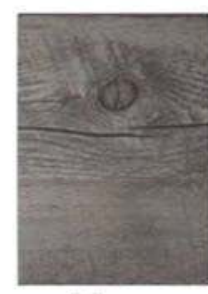

(a)

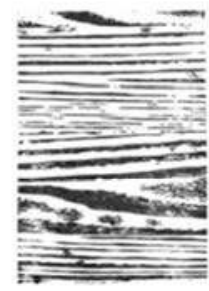

(b)

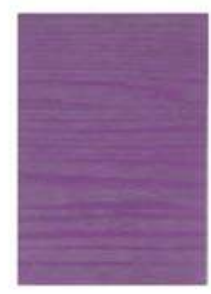

(c)
Fig.2. Picture of sample 3 (a) WC sample; (b) ink stamping of the surface; (c) R sample.

\subsection{Morphological characterization}

Morphological characterization was performed using two complementary procedures. Firstly, a topographical characterization was conducted by means of a profilometer. Secondly, a sensory approach was adopted to qualify the perceived roughness. Note that the central area of each sample was preferentially explored, thus the topographical characterization focused on these central areas. The surface topography of both sets of samples was characterized using a confocal chromatic microscopy sensor from AltiSurf(C) Manutech USD. For time and cost reasons, the whole area of each sample could not be assessed. However, a specific area $\left(12.2 \mathrm{~cm}^{2}\right)$ located in the middle of each sample was defined for consideration. Eighty-one profiles, oriented along the transverse direction, were extracted from these areas and the average value $\left(<R_{q}>\right)$ of the quadratic roughness parameters $\left(R_{\mathrm{q}}\right)$ was calculated. In parallel, a qualitative description of the surface topography was assessed by seven human subjects. First, each person was asked to individually categorize tactile features (explored along the transverse direction). They could identify each sample complemented with the corresponding ink-stamping. The identification was through verbal feedback on how they felt about the texture. Then, these seven subjects were brought together in a group session to work on a consensus description and name for these topographical features based on their individual results.

\subsection{Sensory characterization}

\subsubsection{NappOmatic setup}

The sensory experiment in this study aims at collecting insights on the tactile perception of the two sets of samples. The procedure used here was adapted from Napping® [19] which is a descriptive method derived from the projective mapping [20], originally developed for food and beverages. In projective mapping, assessors are asked to collocate the products on a large sheet of paper, accord- 
ing to their similarities and dissimilarities. Projective mapping serves as a simple and quick technique to obtain product inter-distances. We developed a custom-made setup, "NappOmatic", to perform projective mapping with samples of materials and textures under visual, tactile and visual-tactile conditions [21-22]. The NappOmatic setup is displayed in Figure 3. The mapping area was defined as a square of $93 \mathrm{~cm} \times 93 \mathrm{~cm}$. For tactile tests, the room was dark and the table was equipped with UV back lights that enable to see the sample holder silhouette and position, without seeing the texture and details of the sample surface. In this setup, the assessor could easily perform a tactile exploration of the sample surface and position the sample on the table. The tabletop was made of a translucent material and a camera, installed under the table, took a picture of the mapping surface at the end of each test. The back of the samples were tagged with QR code printed on fluorescent paper to increase the contrast under UV light and to enable the automatic extraction of the sample positions, using software specifically developed for this task. For each assessor $j$, the data collected are the coordinates $X_{i j}$ and $Y_{i j}$ of each sample $i$ and descriptors associated to the samples by the assessors during the experiment. These data were processed by means of a Multiple Factor Analysis (MFA) implemented in SensomineR software [23-24]. MFA is based on a Principal Component Analysis (PCA) and has the advantage of allowing the structuration of the data in group to balance the influence of the assessors in the analysis. From the MFA analysis, for a given sensory modality, a mean representation of the samples among the panel was extracted. On this mean representation, confidence ellipses around the position of each sample could be built. The ellipses represent $95 \%$ of different possible positions that can be taken by a sample [25]. In addition, the descriptors cited by the assessors give indications as to how the samples were perceived. These descriptors also give insights on the meaning of the axes deriving from the MFA.

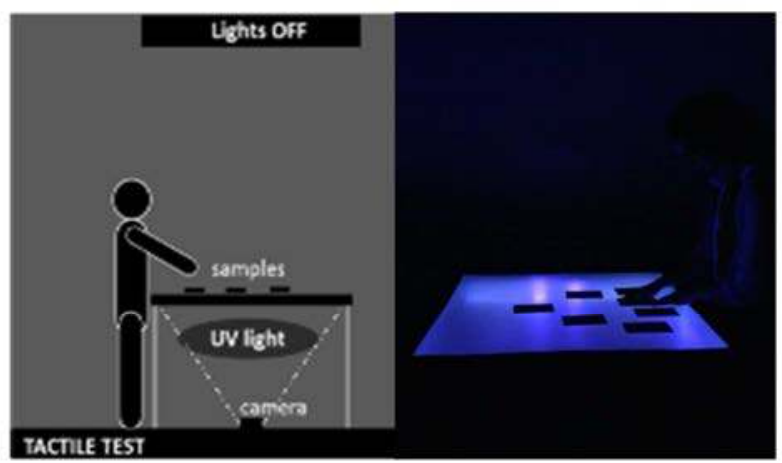

Fig.3. NappOmatic setup for tactile perception of surface textures.

\subsubsection{Test instructions}

The assessors were instructed to arrange the samples on the table, according to their perceived similarities and differences. Samples that are perceived to be similar should be close together, and those that are different should be far from each other. After positioning the samples, the assessors were asked to give attributes, i.e. words that qualify their own perception of the texture of each sample or group of samples. They were not allowed to lift the sample from the table, but the exploration movements unconstrained.

\subsubsection{The assessors}

A panel of 18 untrained people participated in the tactile perception test with each set of samples. The panel consisted of 10 females and 8 males for both sets of samples. It included: master's students, teachers, technicians, administrators and $\mathrm{PhD}$ students. They were all unaware of the purpose of the experiment, and they had never been involved in a sensory test. They were all volunteers, and did not receive any compensation for their participation. They were all recruited within the Ecole des Mines de Saint-Etienne.

\subsection{Tribo-tactile characterization}

The goal was to define measurable features (objective indices) that assist the differentiating the samples in a comparable manner, as in tactile perception tests.

\subsubsection{TriboTouch setup}

The experimental TriboTouch setup [9-11], shown in Fig. 4 , was used. The samples were set on a guide which has translational motion provided by two identical compliant mechanisms, which hold a central base with force transducers and the sample holder. The compliant guides are designed to enable the sample to move up to $100 \mathrm{~mm}$. They consist of two parallelograms. Bearings are replaced by flexible hinges in order to avoid any other sliding contact and to prevent possible parasitic vibrations, which could compromise the measurements of vibrations rising from the contact between the fingertip and the sample surface.

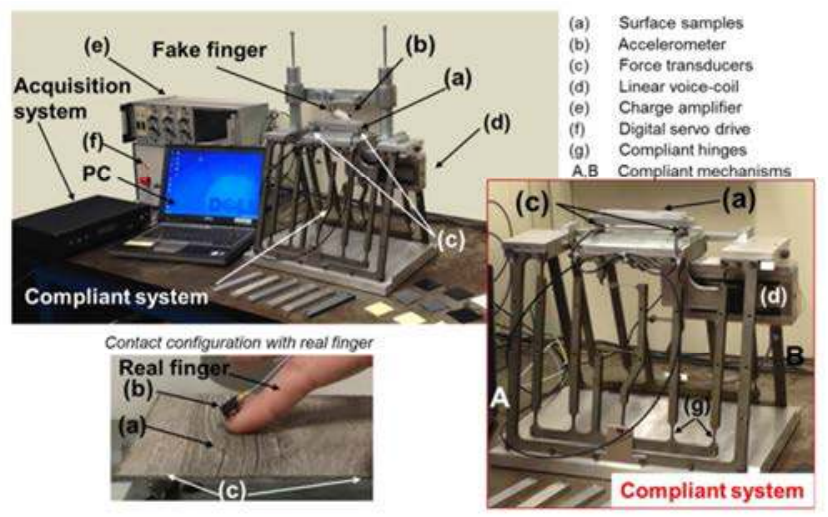

Fig.4. The TriboTouch setup.

The horizontal translation of the sample is obtained, using a linear voice coil actuator controlled by a computer. The sample position feedback is provided by a TransitorTransistor-Logic (TTL) linear encoder that allows for clos- 
ing of the control loop ( $0.5 \mu \mathrm{m}$ of resolution). The TriboTouch setup collects the contact forces and the frictioninduced vibrations produced by the sliding contact between the finger of the operator and the surface of the sample. The forces exchanged between finger and surface are measured by two tri-axial force transducers (Kistler, model 9017B). Normal and tangential forces provide an estimate of the mean friction coefficient during the sliding contact. Before reaching the acquisition system, the detected signals are sent to the charge amplifiers (Kistler, model 5007). An accelerometer is placed on the nail of the operator and secured there with a light wax layer. The accelerometer recovers the vibrations generated by the fingertip as it scans the sample surface. In rare instances when it is not possible to detect the vibrations at the contact interface, the measurement at the fingernail makes it possible to collect the spectrum distribution of the overall induced vibrations.

\subsubsection{Test Conditions}

Each sample of the WC and R sets was positioned on the TriboTouch, and was explored with the right-hand index finger at a constant scanning speed. The finger axis was parallel to the scanning direction during the tests. The finger in contact with the horizontal surface was inclined at an angle of about $20^{\circ}$ (Fig. 5), resulting in application of a normal force of about $0.5 \mathrm{~N}$ throughout the stroking motion (only tests with a variability of maximum $\pm 0.05 \mathrm{~N}$ were accepted). The fingertip was cleaned with an alcohol solution before each measurement and the inclination angle of the finger was ensured by means of appropriate arm support. The acceleration at the fingernail and the forces were measured using an OROS35 data acquisition system, with a sampling frequency of $5 \mathrm{kHz}$. A stroke of $60 \mathrm{~mm}$ with a speed of $20 \mathrm{~mm} / \mathrm{s}$ was set to control the movement of the guide. The region of interest for the test was the center of the sample (starting at $45 \mathrm{~mm}$ from the edge), which was the preferential exploration area during the NappOmatic experiment. A cycle of 2 strokes (outward and return), with constant acceleration and deceleration at the beginning and at the end of the stroke, was set on the test bench. A constant velocity was maintained in the middle of each stroke. Only the signals acquired during the constant velocity phase of the outward stroke were considered for the purposes of this analysis. The strokes analyzed began with the finger at a distance of 55 to $95 \mathrm{~mm}$ from top of the sample $(40 \mathrm{~mm}$ covered in a 2second time lapse).
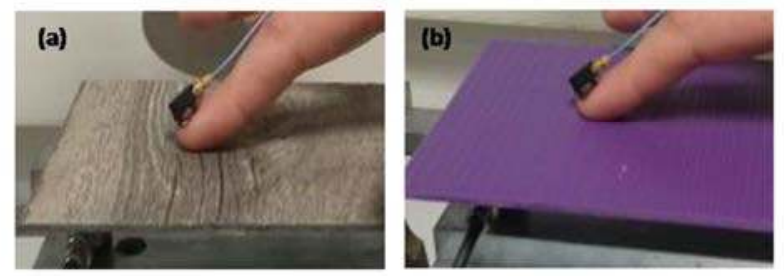

Fig.5. Wood countertype (a) and replica sample (b) on the TriboTouch setup.
The offline-processing was performed using MATLAB to obtain the plot of the parameters of interest and calculate the different indices that could be associated with surface perception (Fig. 6). The tests were replicated at least 3 times in order to verify the consistency of results. Results with low standard deviations were found to be of high repeatability and were kept for the analysis.

\section{Results AND Discussion}

Based on the various approaches used to characterize the two sets of samples, different parameters were extracted. Firstly, the morphological characterization provided the overall roughness of each sample, represented by the mean quadratic roughness $\left\langle R_{q}\right\rangle(\mu \mathrm{m})$, and a qualitative description of topographical features. Secondly, the NappOmatic experiments furnished the mean representation of the tactile perception of the samples. Finally, the TriboTouch tests gave tribological and vibrational indices for each sample. In this section, the complementary data are compared.

\subsection{Morphological analysis}

In order to assess the quality of the replication procedure, the average value $\left(\left\langle R_{q}\right\rangle\right)$ of the quadratic roughness parameters $\left(R_{q}\right)$ obtained from the eighty-one profiles was calculated. Figure $6 \mathrm{~b}$ shows the comparison between $\left\langle\mathrm{R}_{\mathrm{q}}\right\rangle$ values obtained for WC samples and $\mathrm{R}$ samples. The $<\mathrm{R}_{\mathrm{q}}>$ values of all the samples, including $\mathrm{WC}$ and $\mathrm{R}$ sets, are between $4 \mu \mathrm{m}$ and $7 \mu \mathrm{m}$. These measurements confirm that the replication procedure is adequate and that it makes it possible to efficiently replicate the surface topography of the WC samples. Therefore, the surface topographies of WC and R samples are considered to be similar.

As illustrated in Fig. 2b, the surface topography of all the samples is for the most part oriented. In Fig. 6a, a profile extracted from sample 5WC shows that the surface topography is made up of valleys and peaks of different widths that represent the wood grain. The average height $\left\langle\mathrm{H}_{\alpha}\right\rangle$ of the highest peaks and lowest valleys, calculated based on over one hundred values, was found to be about 250 $\mu \mathrm{m}$. In addition, the profile shows that a finer texture is noticeable over the large valleys and large peaks. This fine texture is also oriented as illustrated by the profile (Fig. 6a) and represents the wood fibers. An average height $\left\langle\mathrm{H}_{\beta}>\right.$ of $50 \mu \mathrm{m}$ among the highest peaks and lowest valleys of these sampling areas was calculated based on over one hundred values. Note that all samples exhibit similar features on their surface topography. For both of the texture features (wood grain and wood fibers), the spatial period is greater than $100 \mu \mathrm{m}$, which means that these samples can be considered "coarse" according to the duplex perception mechanism. Fig. 7 depicts a graphical representation highlighting noticeable features of the surface topography of the different samples classified by the seven assessors. The features identified are the result of a combination of the visual aspect and the induced tactile perception. The identified features were classified in four categories. The first, type $C$, perceived as the smoothest, accounts for the areas with large peaks and 
valleys ( $\mathrm{w}_{\mathrm{p}} \geq 4 \mathrm{~mm}$ and/or $\mathrm{w}_{\mathrm{v}} \geq 4 \mathrm{~mm}$ ). The second category (type A), perceived as the roughest, is characterized by alternating narrow valleys $\left(1 \mathrm{~mm} \leq \mathrm{w}_{\mathrm{v}}<2 \mathrm{~mm}\right)$ and peaks $\left(1 \mathrm{~mm} \leq \mathrm{w}_{\mathrm{p}}<2 \mathrm{~mm}\right)$.
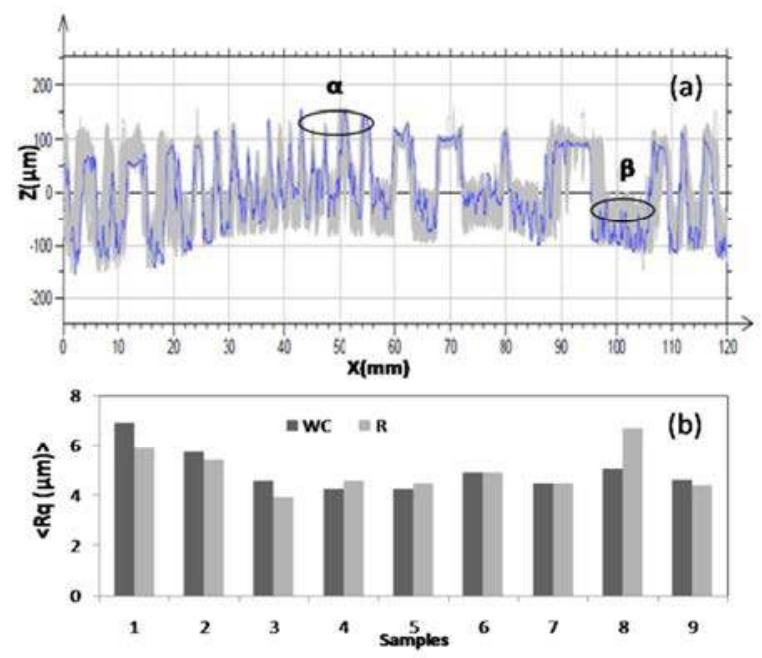

Fig.6. (a) Surface profiles; (b) Histogram representing the overall sample roughness.

A third category (type $\left.A^{\prime}\right)$, characterized by narrow peaks $\left(1 \mathrm{~mm} \leq \mathrm{w}_{\mathrm{p}}<2 \mathrm{~mm}\right)$ in large valleys $\left(4 \mathrm{~mm} \leq \mathrm{w}_{\mathrm{v}}\right)$, was also perceived as rough. The rest of the sample surface was considered "background" (type B). It was characterized by the alternation of both wide valleys $\left(2 \mathrm{~mm} \leq \mathrm{w}_{\mathrm{v}}<\right.$ $4 \mathrm{~mm})$ and peaks $\left(2 \mathrm{~mm} \leq \mathrm{w}_{\mathrm{p}}<4 \mathrm{~mm}\right)$. This "background" was perceived as having a medium roughness. In order to transcribe the tactile perception of each topographical feature, an additional experiment was performed. Covers were added on the surface of the R samples, exposing only a small $4 \times 4 \mathrm{~cm}^{2}$ area of the sample to the assessor. Twelve areas representing all three types were presented. Then an unconstrained sorting task was conducted on these areas using a group of 11 participants. They had to classify the areas according to their perceived "feel", from the smoothest to the roughest. The average ranking, calculated based on all the participants, confirmed the ranking obtained from the consensus group. Based on the consensus group session and the unconstrained sorting task experiment, a graphical representation was proposed, with areas of type B in grey, type A in red, type $A^{\prime}$ in red with blue dashes and type $C$ in blue (Fig. 7). This representation highlights similarities among samples. For instance, Samples 1 and 6 are very similar and the threesome of 2, 4 and 8 exhibits an alternation of red areas (rough) and blue areas (smooth).

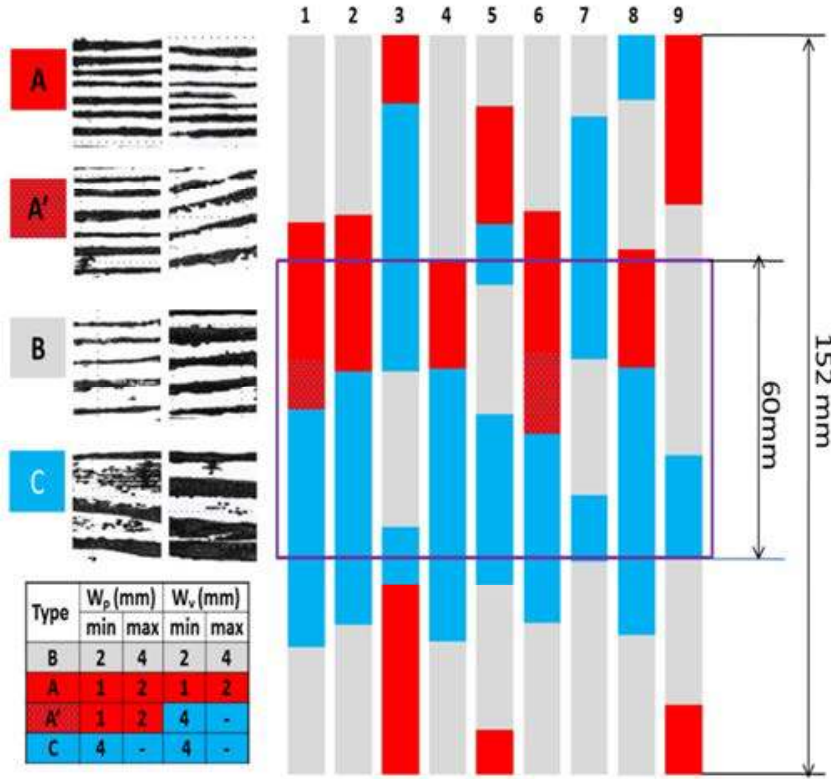

Fig.7. Perceived roughness description along the sample length.

\subsection{Tactile sensory analysis}

From the sensory experiment, mean representations obtained through the MFA are associated with respective confidence ellipses in Fig. 8. The $\mathrm{R}$ map exhibits larger ellipses and a higher degree of overlapping than the WC map, which might indicate that discrimination performances of the assessors for the $\mathrm{R}$ campaign are lower than for the WC campaign. In other words, the samples were perceived to be very similar. Nevertheless, the WC maps allow us to distinguish two groups of samples along dimension 1: on the left there is a group with samples $3 \mathrm{WC}$, $5 \mathrm{WC}$, and $7 \mathrm{WC}$, and on the right is a group with samples 1WC, 2WC, 6WC, 8WC, and 9WC. Sample 4WC lies in between. On the R map, such distinct groups are not visible, however, it can be noted that samples $3 R, 5 R$, and $7 R$ also lie on the left of dimension 1.
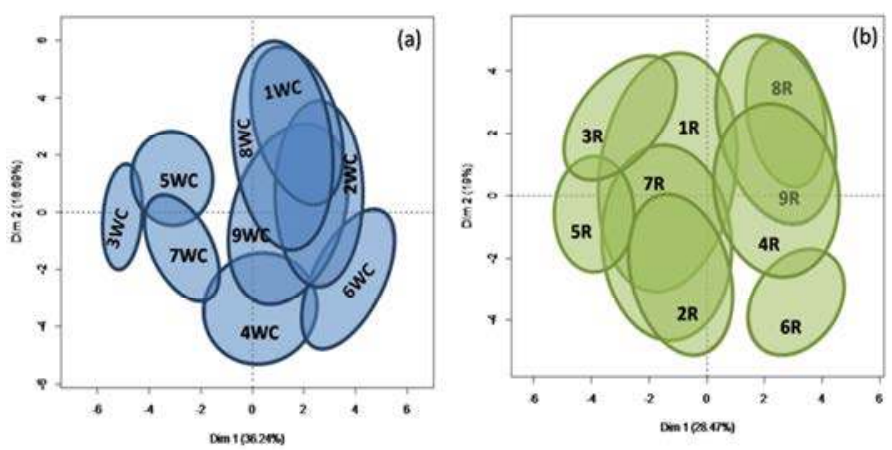

Fig.8. Confidence ellipses around the positions of WC (a) and R (b).

During the tests, assessors provided verbal feedback to qualify the perceived textures, spoken in French and later translated into English. Words with close meanings and synonymous words were combined under a single word (the descriptor) using the dictionary of sensory words 
[26]. For example, the words "rough", "granular" and "not smooth" were put under the descriptor "rough". 14 descriptors from a list of 66 words were defined for the WC campaign, and 9 descriptors from a list of 57 words were defined for the $\mathrm{R}$ campaign. Descriptors frequently used expressed general characteristics of samples that could be used for any texture. Those were rough, grooved, soft and relief. Insights on the meaning of the axes of the mean representation and, therefore, on the discrimination criteria chosen by the assessors during the NappOmatic tests can be obtained based on the mean representations derived from the MFA analysis of the WC and $\mathrm{R}$ campaigns associated with the descriptors provided by the assessors. To address this issue, coordinates on the mean representations were associated with each descriptor. For a given descriptor, the frequency of occurrence was filed for each sample. The descriptor's coordinates were then determined based on their barycentric coordinates, which had been calculated using the coordinates of the sample, weighted in turn by its frequency of occurrence. The map was obtained by projecting these coordinates onto the mean representation, labeled with the associated descriptor (Fig. 9).

The WC descriptor map shows that dimension 1 divides the samples into smooth and rough. This dimension seems to be the most discriminative. Samples 3WC, 5WC and 7WC lie on the left and are perceived as the smoothest. This is consistent with the overall roughness measured using the profilometer (Fig. 6b), according to which those samples do, indeed, exhibit low roughness. In addition, this descriptor map can also be studied in relation to the perceived roughness descriptions depicted in Fig. 7.
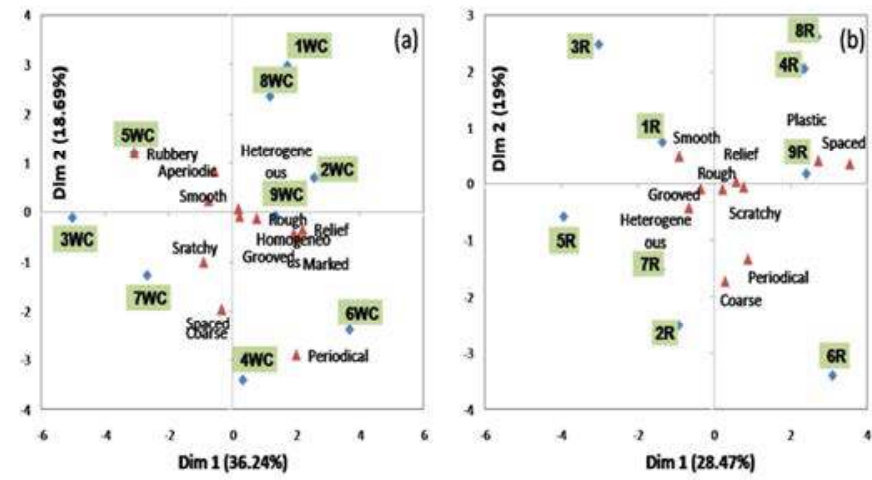

Fig.9. Descriptors map for WC (a) and R (b)

Different combinations of patterns were observed in the central area of all the samples. It appears that the three samples in question ( $3 W C, 5 W C$ and $7 W C$ ) exhibit a sequence of type $B$ followed by type $C$. In the end, this topographical sequence was perceived as the smoothest among the samples provided. Samples 1WC, 2WC, 4WC, $6 \mathrm{WC}$, and $8 \mathrm{WC}$, on the other hand, lie on the right of the descriptor map, and were therefore described as rough. This result was also consistent with the topographical pattern description presented in Fig. 7. These samples exhibit sequences of type A or $\mathrm{A}^{\prime}$ followed by type C. Such sequences highlight the fact that a contrast between a rough pattern $\left(\mathrm{A}, \mathrm{A}^{\prime}\right)$ and a smooth one $(\mathrm{C})$ might give rise to a perception of greater overall roughness.

The descriptors in the $\mathrm{R}$ descriptor map do not allow us to clearly assign meaning to the MFA dimensions. Note that the "smooth" descriptor was still on the left of dimension 1 . The results of the sensory analysis presented were in agreement with the perceptual dimension of tactile texture reported by Okamato et al. in their paper [27]. According to them, roughness/smoothness is one of the three prominent psychophysical dimensions (fundamental dimensions) of tactile texture perception. This dimension was found to be the most discriminating factor for the WC samples.

\subsection{Tribo-tactile analysis}

Using the TriboTouch setup, the accelerometer, placed on the fingernail, makes it possible to acquire the acceleration signal of the vibrations produced by scanning the fingertip over the surface (Fig. 11a). Fig. 10 shows an example of the contact forces and friction coefficient measured during the "touching" phase (9WC) with constant velocity. The normal and tangential forces were almost constant and so was the friction coefficient.

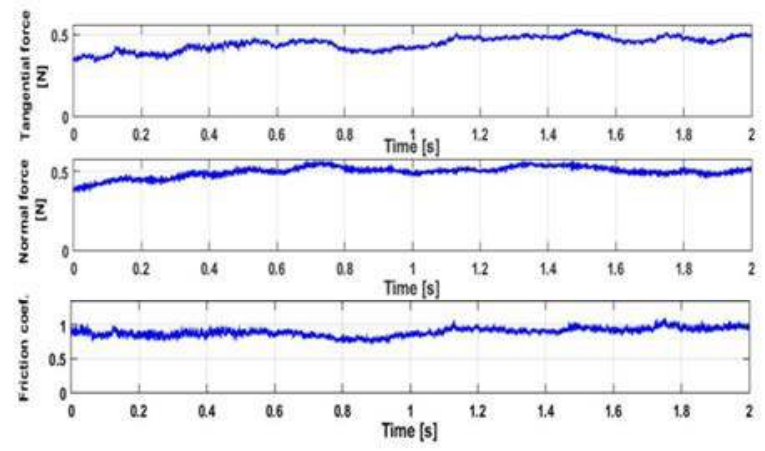

Fig.10. Normal and tangential forces, with the respective friction coefficient, as measured during tactile examination of sample 9WC.

The collected data provided information about characteristic aspects of the touched surfaces, with the aim of defining objective indices to discriminate the samples, thus making it possible to relate those objective indices to the NappOmatic results. Several indices would then be able to be calculated from the tribological and dynamic signals, including the root mean square of the acceleration (accRMS, Fig. 11c), the mean values and standard deviation of the force signals. The acceleration RMS was calculated at time intervals of 0.1 seconds.

\subsubsection{Materials effect}

As expected, for a given set of samples (WC or R), the friction coefficients are very similar between samples in the same campaign $\left(\mu_{\mathrm{WC}}=0.89 \pm 0.068\right.$ and $\mu_{\mathrm{R}}=0.71 \pm$ 0.06). Such behavior is also observed for the RMS acceleration, accRMSWC $=0.61 \pm 0.08 \mathrm{~m}$.s -2 and accRMSR $=0.31 \pm 0.04 \mathrm{~m} . \mathrm{s}-2$. When comparing the same sample numbers from the two different groups ( $\mathrm{R}$ and $\mathrm{WC}$ samples), and thus made of the two different materials, a lower mean friction coefficient (about 20\%) and a lower mean acceleration RMS (about 50\%) were measured for the R samples, as shown in Figure 11. Therefore, based on 
this instrumental vibrational approach, the $\mathrm{R}$ samples are more difficult to differentiate than the WC samples. This result is consistent with the ellipse maps (Fig. 8), and might also confirm that the vibrational cues are used by the assessors to discriminate the samples. Let us consider a given sample number $(9$, for instance) that appears in both groups WC (9WC) and in R (9R), for which we observe that the friction coefficient and RMS acceleration of the R sample are lower than those obtained for the WC sample (Fig. 11). Note that this trend exists across all sample numbers. Considering that the topographical characteristics of the surface are identical, the lower friction coefficients could be directly related to the differences in material properties. To further explore the relationships between tribological indices and sensory results, we will hereafter focus on the WC sample set.
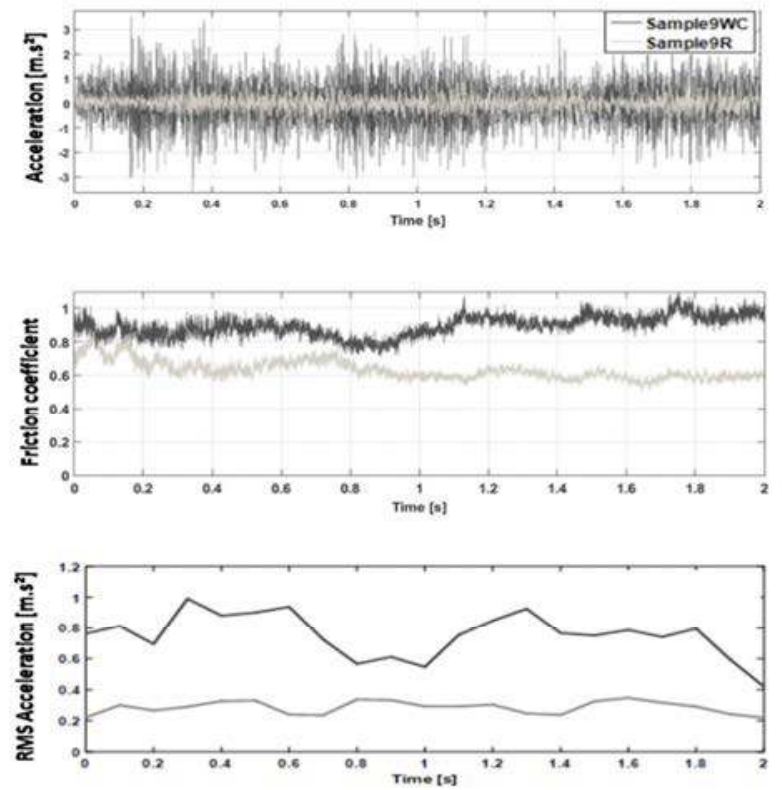

Fig.11. Comparison between the $9 \mathrm{WC}$ and the $9 \mathrm{R}$ acquired acceleration signals (a), friction coefficient (b) and RMS acceleration (c).

3.3.2 Relationship between morphological, sensory and tribotactile approaches

A comparison between morphological, sensory and tribohaptic results was performed. For the WC samples, a strong relationship between tactile perception and vibrational parameters was found.

\section{- Relationship between sensory discrimination along dimension 1 and RMS acceleration index}

On the ellipse and descriptor maps (Fig. 8), dimension 1 carries $36 \%$ of variability and divides the samples into two main groups, the smooth samples on the left and the rough samples on the right. This observation is consistent with the trend observed in the RMS acceleration behavior. The temporal variation of the RMS was then analyzed. In particular, the standard deviations over time were calculated (Fig. 12b). For samples 3WC, 5WC, and 7WC, the standard deviation is below $0.1 \mathrm{~m} \cdot \mathrm{s}^{-2}$ and, as illustrated for 7WC, the RMS acceleration is almost constant. For samples $1 \mathrm{WC}, 2 \mathrm{WC}, 4 \mathrm{WC}, 6 \mathrm{WC}, 8 \mathrm{WC}$, and $9 \mathrm{WC}$, the standard deviation is above $0.1 \mathrm{~m} \cdot \mathrm{s}^{-2}$ and, as illustrated for $6 \mathrm{WC}$, the RMS acceleration shows large variations.
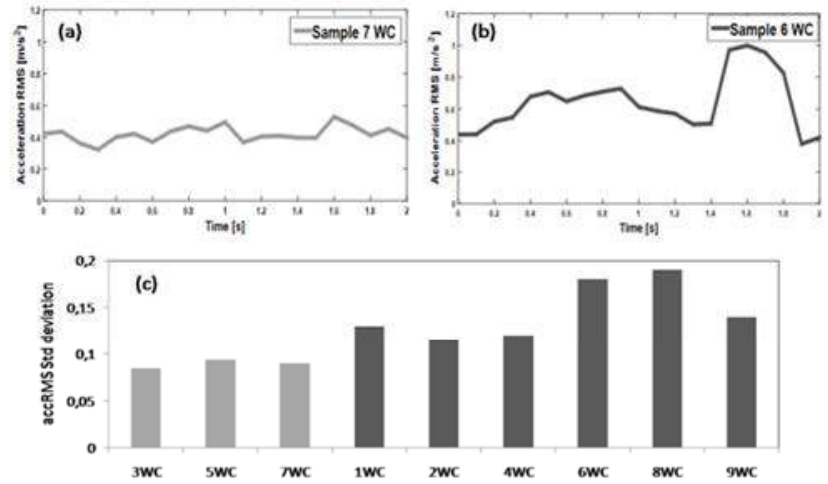

Fig.12. Time variation of the acceleration RMS for samples 7WC (a) and 6WC (b). Standard deviations of the time trend of the acceleration RMS for the samples belonging to the two groups (light and dark gray bars) of WC (c).

This result can be interpreted in terms of smoothness of the surface. Indeed, a low standard deviation of RMS acceleration (Fig. 12c, light grey bars) provides evidence of a low roughness contrast between patterns within the explored surface. On the descriptor map, samples $3 \mathrm{WC}$, $5 \mathrm{WC}$ and 7WC are gathered into a group qualified as "smooth", which was confirmed by the perceived roughness description presented in Fig. 7. On the contrary, high standard deviation of RMS acceleration (Fig. 12c, dark grey bars) bears witness to a high roughness contrast between patterns within the explored surface. Samples $1 \mathrm{WC}, 2 \mathrm{WC}, 4 \mathrm{WC}, 6 \mathrm{WC}$, and $8 \mathrm{WC}$ lie on the right of the descriptor map and are therefore described as rough. This is also consistent with the perceived roughness description presented in Fig. 7. These samples exhibit sequences of type A or $\mathrm{A}^{\prime}$ followed by type C. Such sequences highlight a contrast between a rough pattern $\left(\mathrm{A}, \mathrm{A}^{\prime}\right)$ and a smooth one $(\mathrm{C})$, which might produce a perception of greater overall roughness.

- $\quad$ Relationship between sensory discrimination along dimension 2 and friction coefficient

On the ellipse and descriptor maps (Fig. 8), dimension 2 carries $18 \%$ of variability. This dimension does not clearly discriminate the samples based on descriptors. However, as an attempt to qualify the perceptive meaning of this axis, the sample positions along that axis were compared. To do so, samples with large confidence ellipses oriented along dimension 2, i.e. with large variability of position along that axis, were discarded (2WC, 6WC, $8 \mathrm{WC}$ and $9 \mathrm{WC})$. Note that sample $1 \mathrm{WC}$ exhibits a large confidence ellipse but was kept in this analysis because it is located at the extreme top of the map. As a result, it was samples $1 \mathrm{WC}, 3 \mathrm{WC}, 4 \mathrm{WC}, 5 \mathrm{WC}$ and $7 \mathrm{WC}$ that were considered. Fig. 13 plots the y-coordinates of these samples 
along with the corresponding mean friction coefficient. It appears that the friction coefficient is well correlated to dimension 2, in that it increases along that dimension.

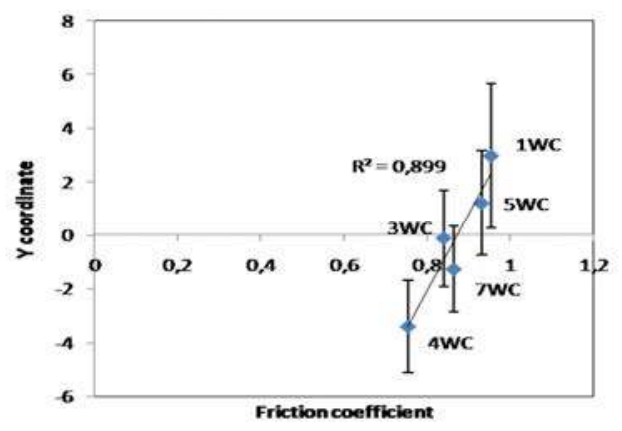

Fig.13. Friction coefficient increasing along dimension 2 ( $\mathrm{Y}$ axis).

\section{Conclusion}

In this study, samples with oriented coarse textures mimicking wood grain and wood fibers were analyzed. They were made of two different materials. Different approaches were used to analyze and discriminate the samples. A sensory setup (NappOmatic) provided maps to represent the tactile perception of the samples, while a tribo-tactile setup (TriboTouch) allowed the sliding contact to be reproduced and permitted the measurement of contact forces, friction coefficient and friction-induced vibrations. The analysis of the acquired signals allowed for the identification of tribological and vibrational indices in agreement with the perceived features of the samples. On the sensory map, the samples were preferentially divided along a smooth-rough axis (x-axis, dimension 1 , WC samples). This axis was found to be related to the RMS acceleration collected using the tribo-haptic setup. In addition, the y-axis of the sensory map was found to be correlated to the value of the friction coefficient.

Comparing the results obtained using the two different materials, a lower friction coefficient was measured for the R set of samples in comparison to the WC samples. In addition, lower discrimination performances were observed during the sensory experiments involving the $\mathrm{R}$ set with respect to the WC. In the WC set of samples, a larger friction coefficient was measured and fairly good discrimination performances were observed during the sensory experiment. This result suggests that the reduction of the induced vibrations caused by a lower friction coefficient contributed to degrading the discrimination between the different samples. The results presented in this work highlight the main role of friction-induced vibrations in the perception of textured surfaces, and suggest the possibility of deriving objective indices that would make it possible to discriminate surface properties in a way comparable to tactile perception. It should be noted that the analysis was performed on samples that are extremely similar to each other from a tactile point of view, and the slight difference is associated with the distribution of the patterns on the overall surface. Nevertheless, even within this particular set of samples, the indices, derived from friction-induced vibrations, were shown to be consistent with the tactile discrimination of the samples.

\section{ACKNOWLEDGMENT}

This work was supported by the LABEX MANUTECHSISE (ANR-10-LABX-0075) of Université de Lyon, within the program "Investissements d'Avenir" (ANR-11-IDEX0007) operated by the French National Research Agency (ANR).

Corresponding author: J. Dacleu Ndengue

(jessica.dacleu@emse.fr)

\section{REFERENCES}

[1] T. A. Whitaker, C. Simões-Franklin, and F. N. Newell, “Vision and touch: Independent or integrated systems for the perception of texture?," Brain Res., vol. 1242, pp. 59-72, 2008

[2] W. M. Bergmann Tiest, "Tactual perception of material properties," Vision Research, vol. 50, no. 24.pp. 2775-2782, 2010.

[3] S. J. Bolanowski, G. a Gescheider, R. T. Verrillo, and C. M. Checkosky, "Four channels mediate the mechanical aspects of touch," J. Acoust. Soc. Am., vol. 84, no. 5, pp. 1680-1694, 1988.

[4] A. M. Fernandes and P. B. Albuquerque, "Tactual perception: A review of experimental variables and procedures," Cognitive Processing, vol. 13, no. 4. pp. 285-301, 2012.

[5] M. Hollins and S. R. Risner, "Evidence for the duplex theory of tactile texture perception," Percept. Psychophys., vol. 62, no. 4, pp. 695-705, 2000.

[6] S. J. Lederman, J. M. Loomis, and D. A. Williams, “The role of vibration in the tactual perception of roughness," Percept. Psychophys., vol. 32, no. 2, pp. 109-116, 1982.

[7] D. E. Sadava, G. Orians, W. K. Purves, H. C. Heller, D. M. Hillis, “Life: The science of Biology 8th edition", Sinauer Associates, Inc.. Figure 45.6, P. 971, 2008.

[8] K. O. Johnson, "The roles and functions of cutaneous mechanoreceptors," Current Opinion in Neurobiology, vol. 11, no. 4. pp. 455-461, 2001

[9] R. Fagiani, F. Massi, E. Chatelet, Y. Berthier, and A. Akay, "Tactile perception by friction-induced vibrations," Tribol.Int., vol. 44, no. 10, pp. 1100-1110, 2011.

[10] H. Zahouani, S. Mezghani, R. Vargiolu, T. Hoc, and M. El Mansori, "Effect of roughness on vibration of human finger during a friction test," Wear vol. 301, no. 1-2, pp. 343-352, 2013.

[11] R. Fagiani, F. Massi, E. Chatelet, J. P. Costes, and Y. Berthier, "Contact of a finger on rigid surfaces and textiles: Friction coefficient and induced vibrations," Tribol. Lett. vol. 48, no. 2, pp. 145-158, 2012.

[12] Hollins M., Bensmaïa S., Risner R.," the duplex theory of tactile perception", Proceedings of fourteenth annual meeting of the international society for psychophysics, 115-120, 1998

[13] Y. Shao, V. Hayward, and Y. Visell, "Spatial patterns of cutaneous vibration during whole-hand haptic interactions.," Proc. Natl. Acad. Sci. U.S. A., p. 201520866, 2016.

[14] H. Culbertson, J. Unwin, and K. J. Kuchenbecker, "Modeling and rendering realistic textures from unconstrained tool-surface interactions," IEEE Trans. Haptics, vol. 7, no. 3, pp. 381-393, 2014.

[15] S. J. Lederman and M. M. Taylor, "Fingertip force, surface geometry, and the perception of roughness by active touch," Percept. Psychophys, vol. 12, no. 5, pp. 401-408, 1972.

[16] Bueno, M. A. , Crest, M., Monteil, G., Berthier, Y.,Lemaire-Semail, B., Massi, F. ,Vinter, A., Aimonetti, J.-M. , Carpentier, L., Cornuault, P.-H. , Giraud, F., Camillieri, B., Ribot-Ciscar, E., Witt, A. ,Gentaz, E., Mith, S., 
“COSTaM: Tool Design for a Controlled Tactile Stimulation", AMSE Journals-Modelling C, pp. 31-42, Vol. 75, n. 2, 2014.

[17] S. Lindberg, A. Roos, A. Kihlstedt, and M. Lindström, “A product semantic study of the influence of the sense of touch on the evaluation of wood-based materials," Mater. Des. vol. 52, pp. 300-307, 2013.

[18] T. Miyashita, T. Maeno, and Y. Nonomura, "How do we recognize biological materials by touch?" Colloids Surfaces B Biointerfaces, vol. 80, no. 2, pp. 176-179, 2010.

[19] J. Pagès, "Direct collection of sensory distances: application to the evaluation of ten white wines of the Loire Valley," Sci. Aliments, vol. 23, no. 5-6, pp. 679-688, 2003.

[20] E. Risvik, J. A. Mcewan, J. S. Colwill, R. Rogersa, and D. H. Lyonb, “PROJECTIVE MAPPING $\square$ : A TOOL FORSENSORY ANALYSIS AND CONSUMER RESEARCH," vol. 5, pp. 263-269, 1994.

[21] P. D. Olivo, B. D. E. L. Curto, J. Faucheu, and D. Lafon, “Sensory Metrology: When Emotions and Experiences Contribute To Design," ICED13 19th Int. Conf. Eng. Des., no. August, pp. 1-10, 2013.

[22] J. Faucheu, A. Caroli, B. Del Curto, and D. Delafosse, "Experimental setup for visual and tactile evaluation of materials and products through Napping ${ }^{\circledR}$ procedure," in International Conference on Engineering Design 2015 (ICED 2015), 2015, vol. in press.

[23] S. Lê and F. Husson, "Sensominer: A package for sensory data analysis," J. Sens. Stud., vol. 23, no. 1, pp. 14-25, 2008.

[24] S. Lê, J. Josse, and F. Husson, “FactoMineR: An R package for multivariate analysis," J Stat Softw, vol. 25, no. 1, pp. 1-18, 2008.

[25] M. Cadoret and F. Husson, "Construction and evaluation of confidence ellipses applied at sensory data," Food Qual. Prefer., vol. 28, no. 1, pp. 106-115, 2013.

[26] F.Bassereau and R.Charvet-Pello, Dictionnaire des mots du sensoriel. Lavoisier, 2011.

[27] S. Okamoto, H. Nagano, and Y. Yamada, "Psychophysical dimensions of tactile perception of textures," IEEE Transactions on Haptics, vol. 6, no. 1. pp. 81-93, 2013.

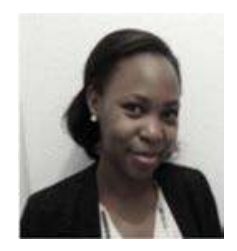

Jessica DACLEU NDENGUE was born in Yaoundé (Cameroon) in 1986. She received a teacher training degree in textile engineering and dressmaking from École Normale Supérieure de l'Enseignement Technique, Douala, Cameroon, in 2007. Then in 2013, an engineering degree in textile engineering and an MS degree in mechanical and fibers science from École nationale d'ingénieurs Sud Alsace, Mulhouse, France. Since December 2013, she has been pursuing a PhD degree in Material Science at École des mines de Saint-Étienne in France. Her PhD project addresses visual and tactile perception of materials' texture and their impact on the perceived quality of products. From 2007 to 2010, she was a teacher at École Normale Supérieure de l'Enseignement Technique, Douala, Cameroon, in the textile industry and dressmaking department.

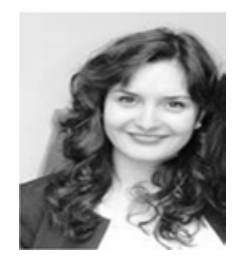

Ilaria CESINI was born in Rome (Italy) in 1991. She received her BS degree in mechanical engineering from the University of Rome "La Sapienza" in 2013. She developed her Master's thesis at the LaMCoS laboratory of the INSA of Lyon, from September 2015 to February 2016, under the Erasmus Plus program. Her project was focused on the analysis of friction-induced vibrations and the tactile perception of textures.
She got her MS degree in mechanical engineering in March 2016. Since October 2016 she has been a part of the Biorobotics PhD program at Scuola Superiore Sant'Anna in Pisa, Italy.

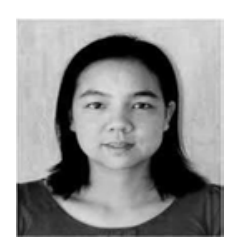

Jenny FAUCHEU graduated in 2002 from the European School of Chemistry, Polymers and Materials (ECPM, Strasbourg). She then joined the National Institute of Standards and Technology (NIST, Gaithersburg, MD), where she spent two years. Back to France in 2005, she defended a PhD in Materials Science at the National Institute of Applied Science (INSA, Lyon) in 2008, on structure-properties relationships in nanocomposite elaborated via latex route. In 2009, she was appointed as Assistant Professor in the École des Mines de Saint-Étienne. Her research focus lies at the interface between materials science and user-centered product design, covering development of new materials for creative industries, materials perception and smart materials.

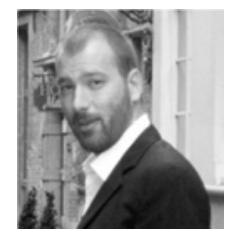

Francesco MASSI received his MS degree at the University of Rome "La Sapienza" in 2003, while he developed his Master's thesis at Carnegie Mellon University of Pittsburgh (PA). In 2006, he simultaneously received a PhD degree in "Theoretical and Applied Mechanics" from the University of Rome "La Sapienza" (Italy) and a PhD degree in "GénieMécanique" from the INSA of Lyon (France). From 2007 to 2013 he was "Maître de Conférences" at the LaMCoS laboratory of the INSA of Lyon. He joined the DIMA (Department of Mechanical and Aerospace Engineering) of the University of Rome "La Sapienza" in the position of associate professor in 2013. His research activities lie in applied mechanics with an emphasis on vibrations, acoustics and tribological contact problems. His research focuses on friction-induced vibrations, addressing friction noise and induced wear of contact surfaces under vibration.

Prof. Massi is a member of the research collaborative network InTriG (International Tribology Group). He is also a member of the ATM (Association de TriboMecanique) society and of the AIT (Associazione Italiana di Tribolgia) society.

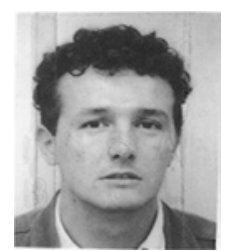

Eric CHATELET received his PhD degree in mechanical engineering from the Franche-Comté University (Besancon, France) in 1994. Since 1997, he has been an assistant professor in the Department of Mechanical Engineering, INSA Lyon, France. His research interests in the Laboratory of Mechanics of Contacts and Structures (LaMCoS, UMR CNRS 5259, INSA Lyon) include the vibrations and dynamics of rotating structures. They focus on damping, on the testing of on board systems, and the dynamics of contacts, with many applications in disc-blades assemblies, in energy transfer and in tactile perception (friction-induced vibrations). 